\title{
Bed capacity and surgical waiting lists: a simulation analysis
}

\author{
Manel Antelo, Universidade de Santiago de Compostela \\ Francisco Reyes Santias, Universidade de Vigo \\ Adela Martinez Calvo, Universidade de Santiago de Compostela
}

\begin{abstract}
Waiting time for elective surgery is a key problem in the current medical world. This paper aims to reproduce, by a Monte Carlo simulation model, the relationship between hospital capacity, inpatient activity, and surgery waiting list size in teaching hospitals. Inpatient activity is simulated by fitting a Normal distribution to real inpatient activity data, and the effect of the number of beds on inpatient activity is modelled with a linear regression model. Analysis is performed with data of the University Multi-Hospital Complex of Santiago de Compostela (Santiago de Compostela, Spain), by considering two scenarios regarding the elastiticity of demand with bed increase. If demand does not grow with an increase on bed capacity, small changes lead to drastic reductions in the waiting lists. However, if demand grows as bed capacity does, adding additional capacity merely makes waiting lists worse.
\end{abstract}

\section{JEL classification}

I10; I12.

\section{Keywords}

Hospital beds; inpatient activity; length of stay; Monte Carlo simulation; surgery waiting lists.

\section{Acknowledgements}

The authors thank two anonymous reviewers for useful comments and suggestions. They also acknowledge discussions with seminar participants at XV Applied Economics Meeting held in A Coruña (Spain) on June 7-8, 2012. MA acknowledges funding from the Galician Regional Government (Xunta de Galicia) through Grant GPC 2013-045. The work of AM-C was supported by the Spanish Ministry of Science and Innovation (Project MTM2008-03010), the Galician Regional Government (PGIDIT07PXIB207031PR), and the Spanish Ministry of Economy and Industry (10MDS207015PR). 


\section{Introduction}

A waiting list for healthcare is a queue of patients who have been given a care procedure but, due to reasons beyond their control, must wait to be served within a variable time period (Sampietro and Espallargues, 2001). Though waiting lists are common in different healthcare fields (both primary and specialty care), healthcare levels (outpatient and hospital care), and therapeutic procedures (surgical and nonsurgical), diagnostic and rehabilitative (Churruca, 2000), surgical waiting lists are those commonly discussed. These are often studied due to their major problems of morbidity and mortality, as well as their greater economic impact (Richards, 1999; Silber et al., 1996).

With the aim of assessing the influence of waiting lists on survival rates, Richards (1999) reviewed 87 studies published in different countries, and showed that survival of patients with over a three-month delay in surgical treatment of breast cancer was reduced by 10 per cent at 5 years when compared to patients who benefited from earlier operations. In the same line, Silber et al. (1996) examined waiting list-related risk, and pointed out that the risk of mortality in patients awaiting coronary by-pass was 1.3 per cent per month, and that by-pass should be performed within the first week after coronary angiography diagnosis to minimize death risks in the waiting list.

Elective surgery in Spain works through waiting lists to such an extent that a public opinion barometer identified waiting time for elective surgery as the leading source of public dissatisfaction with inpatient services. One of the main reasons behind waiting lists for elective surgery is the fact that Spanish hospitals face serious problems of productive capacity (Siciliani and Hurst, 2003). Although productive capacity usually refers to resources such as staff, beds, operating theatres, and community-based health centers, just to name a few, the two main resources in hospital production function are personnel and number of beds (often considered a rough proxy for capital endowment). ${ }^{1}$

This paper is focused on analysing the situation in one of these hospitals, namely the University Multi-Hospital Complex of Santiago de Compostela ${ }^{2}$ (Santiago de Compostela, Spain) Specifically, we examine the impact of bed capacity-a scarce and expensive input in healthcare-on daily inpatient activity, patients' length of stay and, consequently, waiting lists in a teaching hospital like the above mentioned. The high cost of academic health centres and other teaching hospitals is largely attributed to the unique missions pursued by these institutions-including graduate medical education, biomedical research, and the maintenance of standby capacity for highly specialized patients (Commonwealth Found, 1997).

Medicare is often viewed as the primary payer of graduate medical education in view of its size and use of explicit payment adjustments for teaching hospitals (Koening et al., 2003). However, experimentation with the real system would cause a lot of trouble both for patients and staff. For that reason, we are obliged to perform a simulation approach, which represents the system and can be manipulated with no daily healthcare practice disruptions. Indeed, one of the often-mentioned reasons for using simulation as a tool is the experimentation with non-existing systems (Law and Kelton, 1991). Once validated, the simulated model can yield accurate estimates of the behaviour of the real system and help to understand and clarify complex dynamic processes (Yamaguchi et al., 1994). Finally, simulating a process such as admission to elective surgery can also help to identify bottle-neck and congestion points.

\footnotetext{
${ }^{1}$ The common wisdom that the lack of hospital resources other than beds (equipment, for example) may determine waiting lists rather than beds themselves is considered here, by assuming a direct relationship between beds and equipment in such a way that beds represent roughly the amount of capital input of the hospital.

${ }^{2}$ Throughout the paper, we will maintain the Galician name of "Complexo Hospitalario Universitario de Santiago de Compostela" and its CHUS acronym.
} 
Besides, the simulation model can be useful to monitor hospital system performance and assess the relative effectiveness of alternative policies aimed at coping with historical or statistically-generated patient load.

In sum, simulation is a recommended tool to solve the problems created by complex systems where mathematical models are not operational. For this reason simulation is widely used to analyse hospital problems, as these problems are considered a complex system comprising many variables and different random events. For example, in surgical services, Everett (2002) developed a decision support tool to evaluate various policies on surgey waiting lists and bed occupancy. Akkerman and Knip (2004) used simulation to allocate beds to cardiac surgery in order to reduce waiting times. Denton et al. (2007) applied simulation to examine optimal timing of surgery. ${ }^{3}$ Likewise, VanBerkel and Blake (2007) built a discrete event simulation model to evaluate surgical waiting times and support capacity-planning decisions. Although the optimization approach is different in each case, it is aimed at improving all services through optimal resource use.

To examine the pattern of waiting lists size in programmed surgery and reproduce the behaviour of daily inpatient activity, the length of stay and, consequently, waiting lists, a known distribution was fit to each variable. Such distribution then allows us to generate new values for daily inpatient activity and patient length of stay by means of the Monte Carlo method. Finally, after generating new observations of inpatient activity and length of stay, the corresponding simulated waiting list and daily percentage of occupied beds (occupancy rate) can also be created.

In this context, waiting list variations with increased number of hospital beds (a rough proxy for capital input) can also be studied by examining the impact of the number of beds on inpatient activity, length of stay and, consequently, waiting lists. With this purpose, the simulation process was replicated for various increased percentages in the number of available beds in two alternative scenarios. First, the increased number of beds is assumed to lead to no modification of the inpatient activity pattern (no beds effect). Then, alternatively, a change in the number of beds was assumed to modify the behaviour of inpatient activity in the amount given by the inpatient activity-beds elasticity estimated by Kroneman and Siegers (2004) in a comparative study for 10 European countries. All computational programming was performed with free statistical software R.

The current research provides two main results. Firstly, if there is no beds effect, i.e., if the number of beds affects the admission pattern, even remarkable extensions in the mumber of beds lead to no significant difference in hospital occupancy rates or waiting lists. Secondly, if the elasticity of the beds-inpatient activity relationship, which is over 1 (Kroneman and Siegers, 2004), is taken into account, then no significant differences in terms of occupancy rates and surgery waiting lists are observed, as the potential number of beds in the hospital increases. In other words, when elasticity is included in the simulation model, waiting lists are found not to drop when the hospital disposes of a higher amount of physical capital (beds) in its production function.

Our findings suggest that, under certain conditions, the number of hospital beds is the driver of waiting lists in Galician hospitals like CHUS. This, obviously, has a key consequences at both policy and managerial levels: hospital configurations (evaluation of bed usage policies, creation of new services, revision of elective admissions...) allocation plans, new view of measures of performance, etc.

\footnotetext{
${ }^{3}$ In spite of the fact that the use of simulation models in healthcare is not new, a survey on 200 healthcare simulation models proves that their results were implemented in only 16 cases, showing limited acceptance (Wilson, 1981). The primary reason for the healthcare industry reluctance to accept simulation was managerial reluctance to reduce complex healthcare processes to a model representation (Alvarez and Centeno, 1999).
} 
Finally, it is worth to note that our results are close to those of Kroneman and Siegers (2004) and Zeraati et al. (2005), among others. In their analysis of the effect of hospital beds on their use for 10 European countries, Kroneman and Siegers (2004) found that admission rates seem sensitive to bed supply, and show a positive and over-1 elasticity (in particular, 1.44). This means that admission rates are higher in those countries whose hospitals have a high number of beds, but the amount of hospital beds itself seems to have no significant impact on average length of stay. In the same line, Zeraati et al. (2005) suggested that an increase in the number of beds tends to generate additional healthcare demand, either in the form of higher patient admission, longer-treated patients, or some combination of both. This fact reflects the so-called Roemer's law of demand (Shain and Roemer, 1959; Roemer, 1961), indicating that a sudden increase in in the amount of hospital beds in a given country, with no changes in other factors, leads to a sharp increase in usage rates.

The rest of the paper is laid out in four sections. Section 2 outlines the model, while simulation analysis and the main results of the paper are presented in Section 3. The results achieved are discussed in Section 4. Finally, Section 5 concludes the paper.

\section{The model}

\subsection{Data on inpatient activity and length of stay}

Our approach is applied to the University Multi-Hospital Complex of Santiago de Compostela (CHUS), a teaching hospital-complex located in the city of Santiago de Compostela (NW Spain). The CHUS is composed of three hospitals with 1,100 beds, 29 surgical theatres and 705 full-time physician equivalents. In 2008 the number of CHUS-treated inpatients was 34,953 vs. 352,930 inpatient days that correspond to 9,525 first-time visits and 149,406 emergencies. ${ }^{4}$

Our working variables are daily inpatient activity and length of stay for all patients admitted in CHUS in 2007. Inpatient or residential activity is the number of hospital patients who, daily, receives lodging and food as well as treatment divided by the number of beds, The length of stay, on the other hand, refers the number of days that a patient is treated in hospital, including readmissions.

To fit an inpatient activity pattern from real data and select adequate fitting distribution, data were split into two groups: working days (i.e. Monday to Friday, except July, August, and December), and holidays (i.e. Saturdays and Sundays plus July, August and December). The reason behind this splitting is that, as plotted in Figure 1, clear differences in inpatient activity values were observed between working days and holidays.

\footnotetext{
${ }^{4}$ Consellería de Sanidade, Xunta de Galicia (2008), Memoria 2007 Sistema Público de Saúde de Galicia. Available at

www.sergas.es/Publicaciones/DetallePublicacion.aspx?IdPaxina=40008\&IDCatalogo=1732.
} 
Figure 1. Box-plot (left panel) and histogram (right panel) of daily inpatient activity.

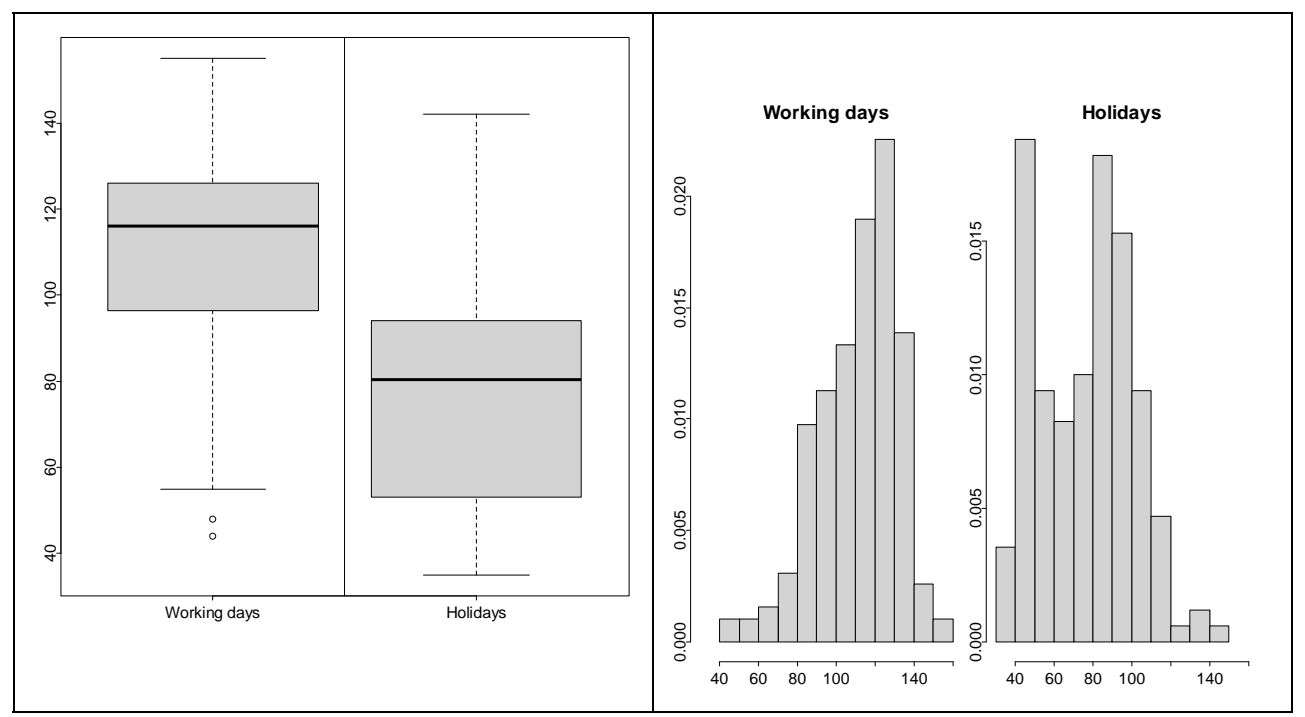

In fact, quartiles are 96.5 (first quartile), 116 (median) and 126 days (third quartile) for working days, while they are 53 (first quartile), 80.5 (median) and 94 days (third quartile) for holidays. A two-sample Kolmogorov-Smirnov test applied to data shows that the statistic value is 0.5667 and the associated p-value is below 0.05 , so the null hypothesis that inpatient activity for working days and holidays is drawn from the same distribution of probability can be rejected. Hence, we decided to analyze inpatient activity in working days and holidays separately.

Both the box-plot and the histogram of patient length of stay are shown in Figure 2. Here, most data can be observed to correspond to short stays, as the first quartile is 3 days, the median is 6 days, and the third quartile is 12 days, although some outliers correspond to longer hospital stays.

Figure 2. Box-plot (left panel) and histogram (right panel) of length of stay.

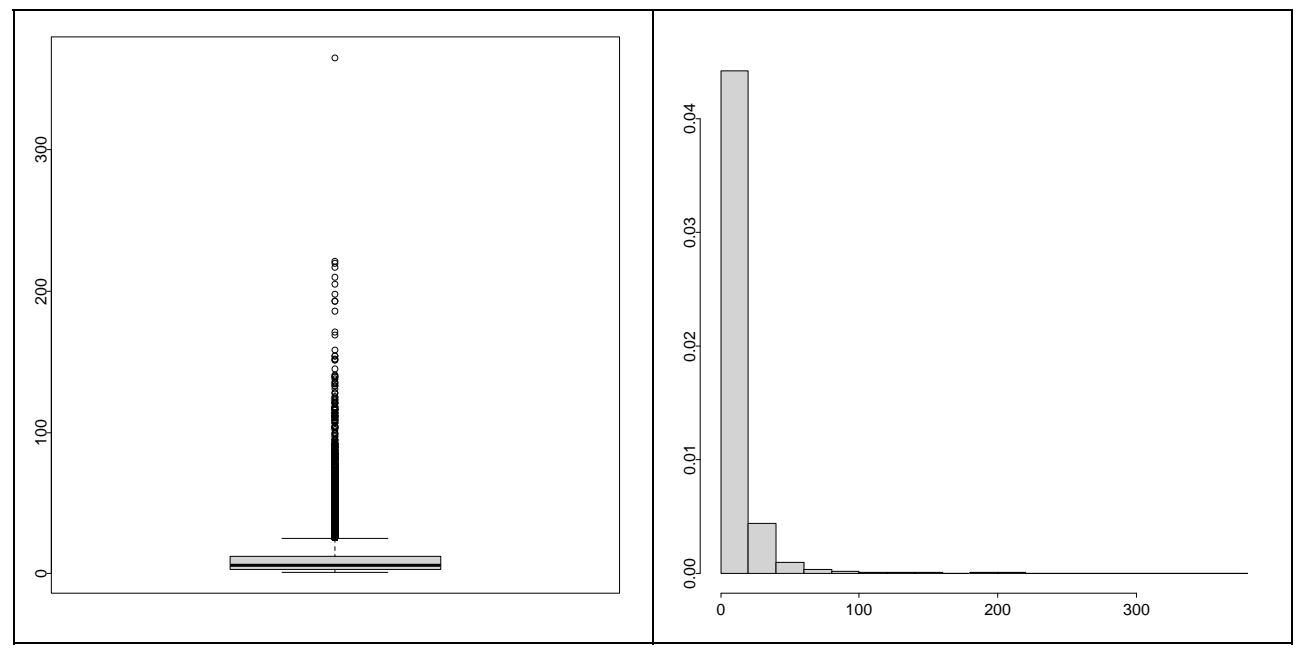

\subsection{The Monte Carlo simulation method}

The Monte Carlo simulation method allows generating new values for daily inpatient activity and patient length of stay. For the former, data were considered independent observations of a continuous variable and then fitted a Normal distribution for 
working days and holidays separately. ${ }^{5}$ In other words, the two parameters (the mean, $\mu$, and the standard deviation, $\sigma$ ) that determine a Normal distribution for working days and holidays were determined. After the fitting procedure, the Normal distribution

$$
N\left(\hat{\mu}_{W}=112.02, \hat{\sigma}_{W}=20.45\right),
$$

was selected for inpatient activity in a working day (denoted by subscript $W$ ), and the Normal distribution

$$
N\left(\hat{\mu}_{H}=76.72, \hat{\sigma}_{H}=24.08\right),
$$

was chosen for inpatient activity in a holiday (denoted by subscript $H$ ). To decide whether the behaviour of the inpatient activity variable comes from a Normal distribution, a Kolmogorov-Smirnov test was completed. This offered 0.0972 as the value of the statistics for working days ( $p$-value $=0.0503$ ), and 0.1047 as the value of the statistics for holidays ( $p$-value $=0.0481$ ). Data are then accepted to be normally distributed for significance level $\alpha=0.01$.

Regarding the length of hospital stay, a Normal and other distributions were fitted like a Poisson distribution, but none of them rendered good fitting. A possible cause could be the presence of outliers corresponding to large stays, which leads the distributions to not have heavy enough tails to fit real data adequately. Hence, an alternative approach based on the kernel density estimator was chosen, for which the length-of-stay variable is implicitly assumed to be continuous.

Denote the real observations of the length-of-stay variable in 2007 by sample $\left(X_{1}, X_{2}, \ldots, X_{n}\right)$ drawn for some distribution with an unknown density $f$, where $n$ represents the sample size. Since we are interested in estimating the shape of the density function of the length-of-stay variable, $f$ can be estimated by the following kernel density estimator (Wand and Jones, 1995)

$$
\hat{f}_{h}(x)=\frac{1}{n} \sum_{i=1}^{n} K\left(x-X_{i}\right)=\frac{1}{n h} \sum_{i=1}^{n} K\left(\frac{x-X_{i}}{h}\right),
$$

where $K$ is a kernel (i.e. a non-negative real-valued integrable function, satisfying the following two requirements:

$$
\int_{-\infty}^{+\infty} K(x) d x=1
$$

and

$$
K(-x)=K(x) \text {, for all values of } x .^{6}
$$

On the other hand, $h$ is a positive number called bandwidth related to the obtained estimator's smoothness. Specifically, the standard Normal density function was adopted as the kernel function, and the bandwidth related to the obtained estimator's smoothness was estimated as $\hat{h}=0.74$.

\footnotetext{
${ }^{5}$ Although we have tried to fit distributions other than Normal distribution, the Normal distribution rendered the best fit.

${ }^{6}$ The first requirement ensures that the kernel density estimation method results in a probability density function, and the second one ensures that the mean of the corresponding distribution equals that of the sample used. $K$ is usually a unimodal symmetric probability density function.
} 


\subsection{Generating new observations}

Once the distributions for daily inpatient activity and patient length of stay were chosen, data were generated for both variables. On one hand, a new observation for inpatient activity is drawn from the Normal distribution stated in (1) on a working day, and from the Normal distribution postulated in (2) on a holiday.

On the other hand, the new observations for patient length of stay were obtained from the kernel estimator given in (3). This was carried out by means of a threestage method. In the first stage, the real length of stay in 2007 was denoted as ( $x_{1}$, $\left.x_{2}, \ldots, x_{n}\right)$, and an element $x$ from dataset $\left\{x_{1}, x_{2}, \ldots, x_{n}\right\}$ was randomly selected. In the second stage, $z$ was generated from the standard Normal distribution $z \rightarrow N(0,1)$. Finally, in the third stage, a new length of stay, $x^{*}$, was built by using the rule

$$
x^{*}=\operatorname{round}(x+\hat{h} z)
$$

where $\hat{h}$ stands for the bandwidth estimated for the kernel density estimator (3), and round $(\cdot)$ rounds the simulated values $x^{*}$ to zero decimal places.

\section{Simulating inpatient activity and patient length of stay}

The activity of the CHUS can now be simulated for 2007, when the total number of beds (roughly speaking, the amount of physical capital) was 1,100. To avoid starting with an "empty" system (i.e. a hospital without patients), the simulation process begins on August $1^{\text {st }}, 2006$ (i.e. before the period of analysis). ${ }^{7}$ Then, for each day of 2007, the simulation process followed a three-stage procedure. In the first stage, an inpatient activity value was generated taking into account both new and waitinglist patients. In the second stage, we detect the number of free beds in the hospital and we decide to occupy them with patients for which generate the length of their stays. Finally, in the third stage, if daily inpatient activity exceeds the number of available beds, the remaining patients are included in the waiting list.

The results of this 3-step model are depicted in Figure 3, which plots the hospital's actual inpatient activity in 2007 (black line), and the 500 runs of model-simulated inpatient activity (grey lines).

\footnotetext{
${ }^{7}$ In the simulation literature, this is called the start-up problem (Law, 1983).
} 
Figure 3. Actual inpatient activity (solid black line) and simulated inpatient activity (grey lines) in 2007.

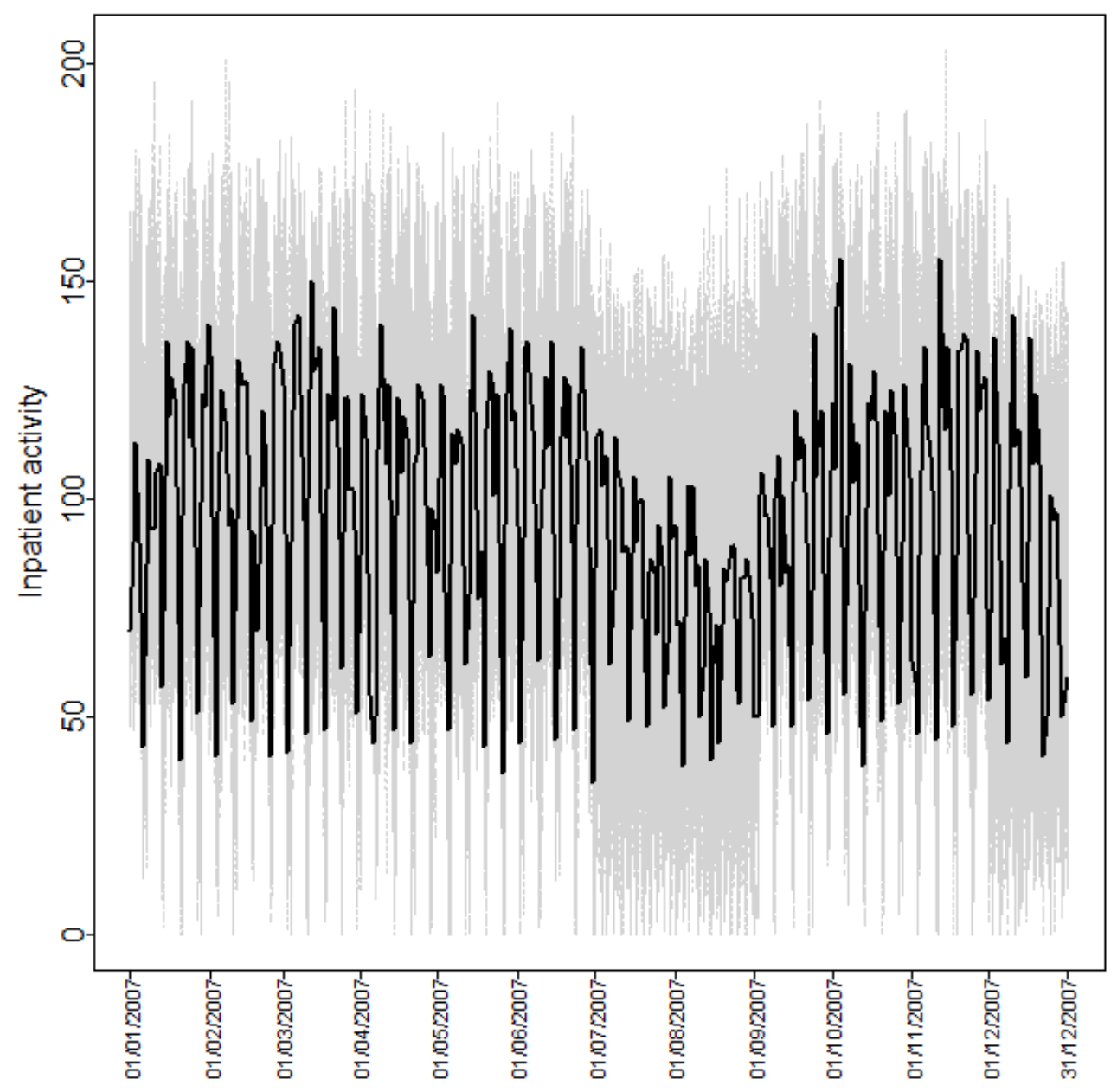

On the other hand, Figures 4 and 5 depict, respectively, the 500 replications of simulated daily waiting lists and occupancy rate. 
Figure 4. Simulated daily waiting list (grey lines) in 2007

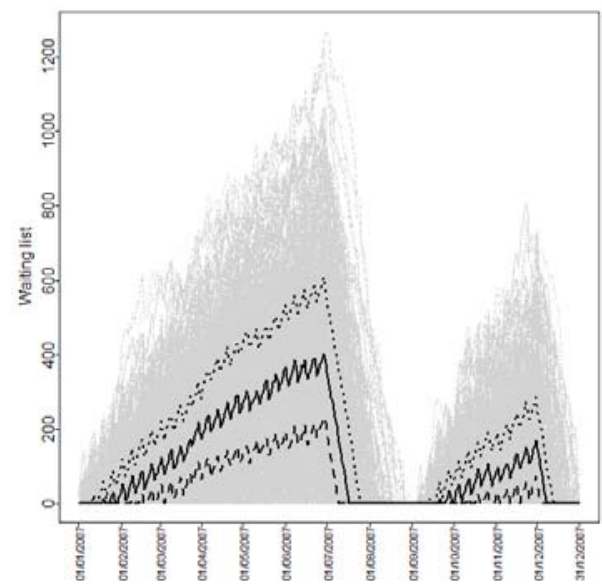

Waiting list quartiles are highlighted: 25 per cent (dashed black line), 50 per cent (solid black line) and 75 per cent (dotted black line).
Figure 5. Simulated daily occupancy

rate (grey lines) in 2007

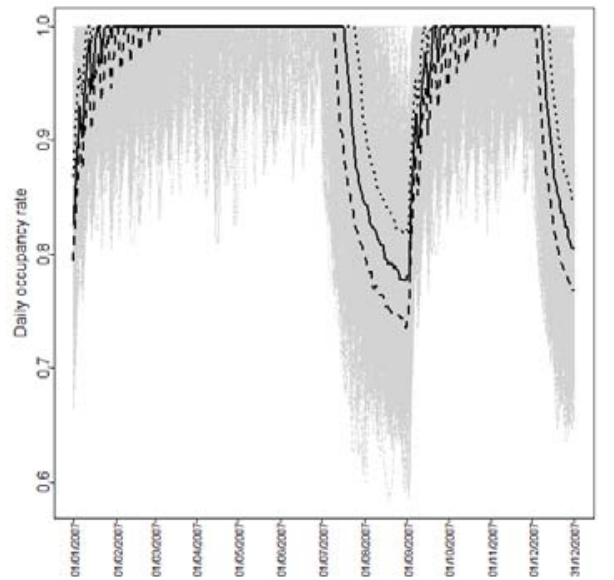

Occupancy rate quartiles are highlighted: 25 per cent (dashed black line), 50 per cent (solid black line), and 75 per cent (dotted black line).

The simulated values can be concluded to fit well the real-data pattern for this period. Besides, the hospital waiting list grows until June, disappears in July and August, and rises again until December. There are then two critical moments before summer and Christmas with very high peaks. A similar pattern is observed for daily occupancy rate.

The results obtained for the simulated daily waiting list data and the simulated daily occupancy rate can be summarized as follows. Thirty-eight days out of a hundred days a year show no waiting list, while 62 days show a variable-size surgical waiting list. This size ranges from 1 to 100 patients (21 per 100 days), from 100 to 200 patients (14 per cent of the analysed period), from 200 to 350 patients (13 per cent of the time considered), and over 350 patients (14 per cent of the time). The differences observed in waiting list time and size would be explained by the seasonal trend observed in hospital admissions. For instance, the admissions of chronicdisease patients in autumn has a strong impact on hospitals' waiting lists, as well as the appearance of flu outbreaks from November to January. Likewise, the impact of spring on some patients with respiratory or allergic disorders can also explain these waiting list differences.

On the other hand, the simulation allows us to conclude that, bed occupation rate ranges between 70 and 80 per cent of hospital capacity in 7 out of 100 days, during 12 per cent of the days this rate ranges between 80 and 90 per cent, and during 19 per cent of the days it ranges between 90 and 99 per cent. That is, 28 out of 100 days the hospital is working below its capacity, while 62 out of 100 days the hospital generates a widely variable-length waiting list. Thus, wide variability is observed in the use of hospital capacity. The fact that bed occupancy rate shows a strong seasonal behaviour may be due to increased healthcare pressure in some months due to the impact of some climate conditions on certain disorders.

\subsection{Beds-inpatient activity elasticity}

In this subsection we examine what happens in the simulation analysis if the number of hospital beds increases. To this end, two alternative scenarios are assumed. First, in Scenario 1 the number of beds has no impact on inpatient activity and the stays pattern. This assumption is then removed in Scenario 2. In both cases, we consider the actual number of beds $(1,100)$, as well as successive increases: 55 beds more 
( $5 \%$ capacity increase), 110 beds more (10\%), 165 beds more (15\%), 220 beds more (20\%), 330 beds more (30\%), 440 beds more (40\%) and 550 beds more (50\%).

\subsubsection{Scenario 1}

Here daily inpatient activity is simulated as described above, i.e. $\hat{\mu}_{W}$ and $\hat{\sigma}_{W}\left(\hat{\mu}_{H}\right.$ and $\hat{\sigma}_{H}$ ) are estimated using the inpatient activity sample of working days (holidays). This implies that no increase in the number of hospital beds, irrespective of the magnitude of the increase, modifies the generation process of new values for inpatient activity or length of stay. In this context, Figures 6 and 7 show, respectively, both the median of the 500 simulated waiting lists and the median of the 500 simulated occupancy rates for various amounts of beds.

Figure 6. Median of simulated waiting lists. No beds effect

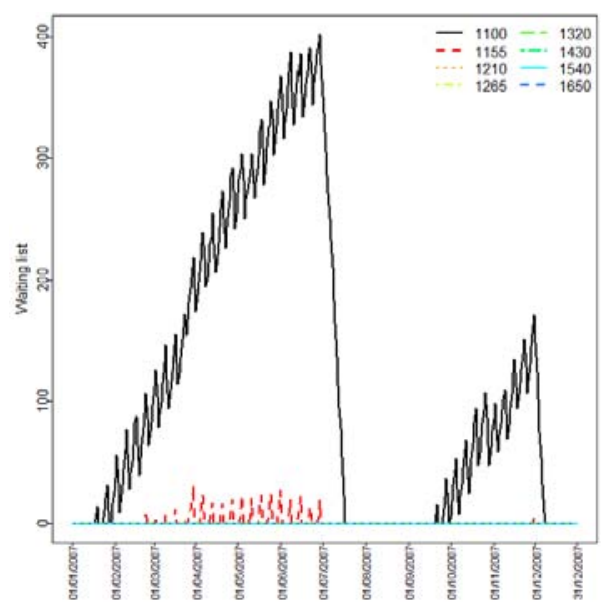

Figure 7. Median of simulated daily occupancy rate. No beds effect

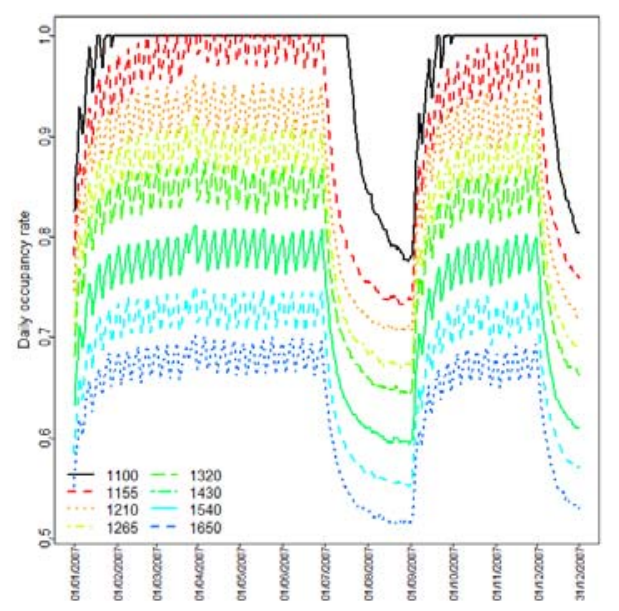

As shown in Figure 6, increased number of beds, although slightly increased, would lead to significantly reduced waiting lists. For instance, a 5 per cent increase in the number of available beds would double the period with no waiting lists (from 38 to 76 per cent). Besides, the remainding waiting list would also be considerably smaller. Particularly, waiting lists comprising 1-100 patients would prevail only 18 per cent of the time (vs. 21 per cent), 100-200 patients would correspond to 5 per cent of the time (vs. 14 per cent), and those comprising over 200 patients would disappear. If the number of beds increased by 10 per cent, the period with no waiting list would extend up to 95 per cent, as there would be a below-100 waiting list during 4 per cent of the time. Finally, if the increase in the number of beds were 15 per cent, there would be no days with waiting lists in the analysed period. In short, a below 15 per cent increase in hospital production capacity would be enough to eliminate waiting lists.

The data shown by Figure 7 allow deducing that a 5 per cent increase in the number of hospital beds would lead to: (i) the number of days of full hospital occupancy (therefore involving waiting lists) would be reduced from 62 to 25 per cent; and (ii) extended periods in which the occupancy rate is below 100 per cent. At the same time, a 10 per cent increase in the number of beds would reduce the number of days in which hospital occupancy rate is 100 per cent up to only 5 per cent per year and would significantly increase the number of days in which occupancy rate remains below 100 per cent. For instance, while the current number of beds leads to hospital occupancy rates between 90 and 99 per cent during 19 per cent of the year, this occupancy rate would extend up to 40 or 42 per cent with either a 5 or a 10 per cent increase in the number of beds, respectively. If the number of hospital beds is increased by 20 per cent, maximum occupancy would not exceed 99 per cent 
throughout the year, while a 30 or a 40 per cent increase would lead maximum occupancy not to exceed 90 or 70 per cent.

The results obtained under Scenario 1 can be formally recorded as follows.

Proposition 1. If bed capacity has no impact on inpatient activity, even a minor increase in the number of beds leads waiting lists to decrease, and occupancy rate to drastically reduce.

\subsubsection{Scenario 2}

In this case we take the median of daily inpatient activity in working days (holidays) and the number of beds in working days (holidays) to fit a linear regression. It is well known that, if the number of hospital beds increases, inpatient activity tends to adapt itself to the hospital's new productive capacity. This is the so-called Roemer's law of demand (Shine and Roemer, 1959; Roemer, 1961). In our case, this effect between capacity and utilization is taken into account by adopting the value that Kroneman and Siegers (2004) obtained for a set of hospitals from 19 European countries. These authors found that a 1 per cent increase in the number of beds leads inpatient activity to increase 1.44 per cent. Thus, inpatient activity seems sensitive to bed supply. This value is taken for the simulations completed in Scenario 2 with the analysed hospital.

Since we are modeling inpatient activity as a Normal distribution, the pattern of the variable is determined by mean and standard deviation. Then we examine whether both parameters, mean and standard deviation, are related to the number of beds. To avoid pernicious effects from outliers, median and median absolute deviation (mad) are considered as a robust estimator of dispersion. ${ }^{8}$

The fitted regression model, where the covariate is the number of hospital beds and the response is the median of daily inpatient activity, is given by

$$
\text { Median (inpatient activity) }=\left\{\begin{array}{l}
3.9619+0.0972 B \text {, in working days } \\
4.2362+0.0643 B \text {, in holidays }
\end{array}\right.
$$

where $B$ denotes the number of beds. Hence, there is a reasonable linear relationship between the median of inpatient activity and beds. Indeed, the determination coefficients for both fittings are over 0.9.

In addition, the fitted regression models, where the covariate is the number of hospital beds and the response is the mad of daily inpatient activity, is

$$
\text { Mad (inpatient activity) }=\left\{\begin{array}{l}
2.3747+0.0165 B \text {, in working days } \\
2.2984+0.0181 B \text {, in holidays }
\end{array}\right.
$$

where, once more, a reasonable linear relationship can be observed between mad of inpatient activity and the number of beds. The determination coefficients for both fittings are over 90 per cent. Therefore, we can use a modified simulation process where the inpatient activity values for working days are drawn from the Normal distribution $N\left(\hat{\mu}_{W}, \hat{\sigma}_{W}\right)$, where

$\hat{\mu}_{W}=3.9619+0.0972 B$ and $\hat{\sigma}_{W}=2.3747+0.0165 B$

\footnotetext{
${ }^{8}$ The mad is the median of the absolute deviations from the data's median. For example, for a dataset as $\{2,2,3,4,12\}$, the median is 3 , so the absolute deviations from the median are $\{1,1,0,1,9\}$ (reordered as $\{0,1,1,1,9\})$ with a median of 1 , in this case unaffected by the value of the outlier 12 . Hence, the mad is 1 .
} 
In turn, inpatient activity values for holidays are drawn from the Normal distribution $N\left(\hat{\mu}_{H}, \hat{\sigma}_{H}\right)$, where

$$
\hat{\mu}_{H}=4.2362+0.0643 B \text { and } \hat{\sigma}_{H}=2.2984+0.0181 B
$$

Figures 8 and 9 sum up the main results provided by the validated model in Scenario 2.

Figure 8. Median of simulated waiting list with a beds effect

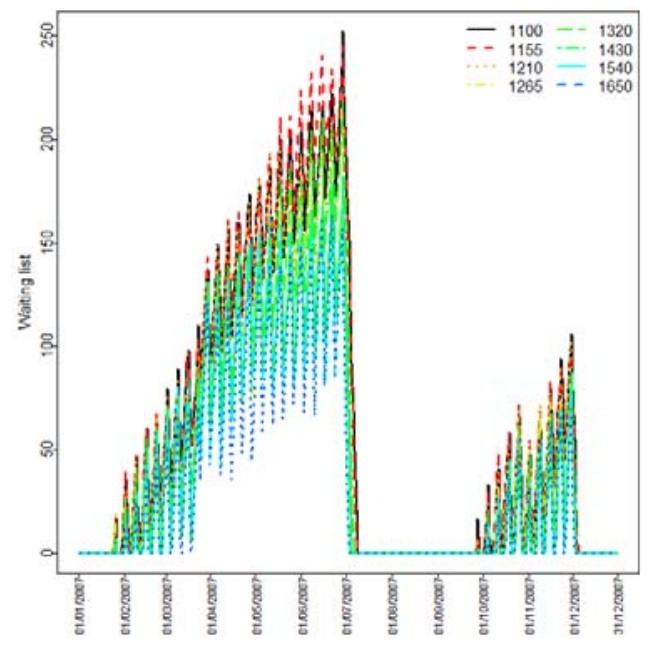

Figure 9. Median of simulated daily occupancy rate with a beds effect

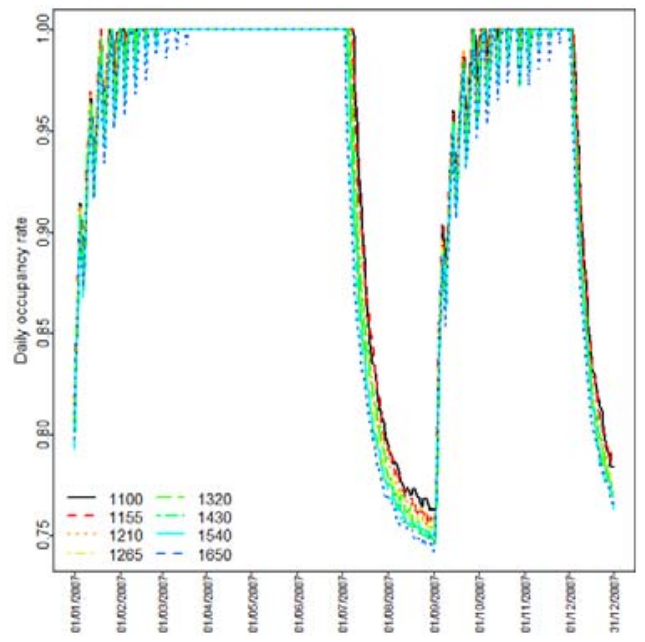

Figure 8 shows that now waiting lists cannot be eliminated, not even when the increase in the number of beds is high. In this scenario in which the admission rate adjusts itself to the number of available beds, a 5 per cent increase in the number of beds is observed not to reduce but to increase waiting lists. From here onwards, waiting lists are reduced if the number of beds keeps growing over 5 per cent. Although the reduction is not proportional, every 5 per cent increase in the number of beds can be said to lead to a 1 per cent waiting list reduction.

Waiting list increases are due to the fact that an increase in the hospital's inputs leads to an increased number of patients included for admission. Besides, given that the elasticity is positive and over-1, greater input amounts lead to greater demand and therefore to longer waiting lists. Though consecutive increases in the number of beds do not shorten waiting lists at the same pace, they do show some effect in this sense, probably due to the appearance of growing scale performances from a particular hospital size which compensate for the effect of (over-1) admissiondemand elasticity relative to inputs.

The simulation results achieved in Scenario 2 can be summarized in the following proposition.

Proposition 2. If we consider the beds effect on inpatient activity, an increase in the number of beds leads to no significant differences in waiting list and occupancy rate (even for huge increases of beds).

\section{Discussion}

The expansion of hospital physical capacity (building new surgical units, for example) is a long-run policy that may require time to be implemented. The increase of the health workforce may be even slower, since physicians and specialists need several years' training before becoming active. Although staff can be recruited from abroad, such staff may face assimilation difficulties and such a policy can therefore 
also take time. This means that the different ways of increasing supply will generally involve different costs and require different time scales. In the short run, purchasing extra activity from public facilities at low marginal cost may be possible if there is spare capacity. However, if public facilities are already working close to full capacity, purchasing extra activity in the short run will only be possible at high marginal costs. In the medium to longer term, it may well be cheaper to expand activity by expanding public capacity. For example, Denmark adjusted its public capacity to respond to the upsurge in demand for coronary revascularisation procedures more rapidly than England in the 1990s. Consequently, waiting times for revascularisation fell in Denmark, whereas they rose steeply in England (Siciliani and Hurst, 2003).

It is argued that, in principle, waiting times can be reduced through supply-side policies, if the volume of surgery is not considered adequate, or through demandside policies, if the volume of surgery is considered adequate. Supply-side policies include raising production capacity by increasing the number of beds and specialists, or by using the available capacity in other (private) hospitals. They also include increasing productivity by funding extra activity, fostering day-surgery, and linking doctors' and hospitals' remuneration system to their performance (Hurst and Siciliani, 2006).

However, it is common to take measures aimed at reducing waiting times by increasing activity, and then find that, after a brief period, demand has increased and waiting times have reverted to levels similar to those before the application of measures. Such responses may be hard to overcome, since demand responds positively to reductions in waiting times. This is the result rendered by the present study. By means of simulation analysis, we observed that if the demand pattern is not modified relative to the increased number of beds, a (slight) increase in the number of beds would remarkably help to reduce surgical waiting lists. Particularly, a 10 per cent increase in the number of available beds would reduce variable-size surgical waiting lists from 62 to 5 per cent of the studied period. In this context, this production capacity increase would be highly effective. However, if the demand responds positively to increased supply, then every increase in the number of beds (no matter how large) would have scarce impact on waiting list reduction.

The outflow (supply) of elective surgery depends on both public and private surgical capacity, and the productivity with which capacity is used. Econometric evidence (cross-sectional and at national level) suggests that higher capacity -in terms of increased numbers of beds and physicians - is associated with lower waiting times. Evidence on the impact of capacity is provided by Martin and Smith (1999) through an English database from the Hospital Episode Statistics in 1991/92. These authors showed that waiting time is negatively associated with the number of available beds. They particularly found that elasticity equals -0.242 . Similarly, Lindsay and Feigenbaum (1984) found out that waiting times are negatively associated to both the number of available doctors and beds.

Furthermore, Álvarez and Centeno (1999) used a simulation analysis for the Washington Adventist Hospital to evaluate the impact of an expansion in the number of beds in the Emergency Room. An expansion in the number of beds was found to result in a 0.6-hour reduction of average length of stay. Kirtland et al. (1995) used simulation to improve performance by reducing patient time in the system and determining appropriate staffing levels. Eleven alternatives were studied and resulted in a reduction of 38 minutes on average.

However, larger increases in capacity may have a different impact on waiting times according to the level of excess demand and initial waiting time. Countries with low supply and high initial waiting times are likely to have elastic demand to waiting-time variations. For this reason, the effect of even larger increases in capacity on waiting times may be rather modest (Hurst and Siciliani, 2006).

In general, supply-side policies may well succeed in their aim of raising the rate of elective surgery, but they may be disappointing in their effects on waiting times. That 
is because an increase in supply may follow rather than lead an increase in demand, or may be overtaken by fresh increases in demand. Moreover, any reduction in waiting times may encourage an increase in admission rates to lists due to lowered clinical thresholds (Hurst and Siciliani, 2006).

Moreover, many commentators suggested that an increase in the supply of hospital beds tends to generate additional demand either in the form of more admitted patients or patients treated for longer time periods, or some combination of both (Zeraati et al., 2005). Formerly, Shain and Roemer (1959) found very close correlations between the availability of short term general hospital beds per 1,000 population, and rates of use as measured by hospital days per 1,000 population. Later, Roemer (1961) also reported on a natural experiment where a sudden increase in hospital beds in a country - with no changes in other factors-led to a sharply increased utilization rates.

Using data from 10 European countries hospitals, Kroneman and Siegers (2004) found out that inpatient activity increases 1.44 per cent for every 1 per cent increase in the number of hospital beds. Taking into account this beds-inpatient activity elasticity, the results regarding the impact of beds on waiting times offered by our study widely differ from those obtained without considering the impact of bed capacity on inpatient activity. Now, an increase in bed capacity leads to no significant differences in waiting lists and occupancy rates, even for huge bed-capacity increases. Our simulation model then reveals the small effect that supply policies may have on waiting lists when healthcare demand adapts itself to the new supply conditions.

\section{Concluding remarks}

Waiting time for elective surgery is a significant problem in the current medical world. This paper aims at reproducing, by means of a Monte Carlo simulation, how the number of beds (a rough measure of a hospital's physical capital) affects inpatient activity, length of stay and, consequently, waiting lists in teaching hospitals like the University Multi-Hospital Complex of Santiago de Compostela (CHUS). Inpatient activity is simulated by fitting a Normal distribution to the real impatient activity data observed in 2007 and the effect of the number of beds on inpatient activity is modelled with a linear regression model. Analysis is performed firstly assuming that the number of beds has no impact on inpatient activity or length of stay (absence of beds capacity effect), and then assuming that the number of beds has certain impact on inpatient activity by adapting itself to the new supply conditions.

This research allows us to evaluate drops in waiting lists due to a potential increase in the number of beds. We add empirical evidence showing that if the effect of the number of beds on hospital admission rates is neglected, then an increase in the number on hospital beds, even the slightest increase, drastically reduces waiting lists. By contrast, if the hospital's admission patterns become affected by the number of beds, every extension in the number of beds, even remarkable extensions, leads to no significant differences in hospital occupancy rates or waiting lists.

\section{References}

Akkerman, Renzo and Marrig Knip (2004) 'Reallocating of beds to reduce waiting time for cardiac surgery', Health Care Management Science 7(2): 119-126.

Alvarez, Adriana M. and Martha Centeno (1999) Enhancing simulation models for emergency rooms using VBA, Winter Simulation Conference, 1685-1693.

Churruca, Silvia (2000) 'Los líderes en gestión de listas de espera advierten: un suceso no puede condicionar la estrategia', Diario Médico, 15/06/2000. Available at http://www.diariomedico.com/sanidad/listas/debate.html (last accessed September 2000). 
Commonwealth Fund (1997), Leveling the Playing Field: Financing theMissions of Academic Health Centers, Report of the Commonwealth Fund Task Force on Academic Health Centers New York: Commonwealth Fund, May.

Denton, Brian, James Viapiano and Andrea Vogl (2007) 'Optimization of surgery sequencing and scheduling decisions under uncertainty', Health Care Management Science 10(1): 13-24.

Everett, Jeffrey E. (2002) 'A decision support simulation model for the management of an elective surgery waiting system', Health Care Management Science 5(2): 8995.

Hurst, Jeremy and Luigi Siciliani (2003) Tackling excessive waiting times for elective surgery: a comparison of policies in twelve OECD countries, OECD Health Working Paper, No. 6.

Kirtland, Anthony, Karen Poisker, Linda Stamp and Patricia Wolfe (1995), Simulating an ED 'is as much fun as?', Proceedings of Winter Simulation Conference, C. Alexopoulos, K. Kang, W.R. Lilegdon and D. Goldsman (eds.), Institute of Electrical and Electronics Engineers, Washington, 1039-1042

Koenig, Lane, Allen Dobson, Silver Ho, Jonathan M. Siegel, Daniel S. Blumenthal and Joel S. Weissman (2003) 'Estimating The Mission-Related Costs Of Teaching Hospitals', Health Affairs 22(6): 112-122.

Kroneman, Madelon and Jacques J. Siegers (2004) 'The effect of hospital bed reduction on the use of beds: A comparative study of 10 European countries', Social Science \& Medicine 59(8): 1731-1740.

Lapierre, Sophie D. and Angel B. Ruiz (2007) 'Scheduling logistic activities to improve hospital supply systems', Computers and Operations Research 34(3): 624641.

Law, Averill M. (1983) 'Statistical analysis of simulation output data', Operations Research 31(6): 983-1029.

Law, Averill M. and W. David Kelton (1991) Simulation Modelling and Analysis, McGraw-Hill, New York, 2nd ed.

Lindsay, Cotton M. and Bernard Feigenbaum (1984) 'Rationing by waiting lists', American Economic Review 74(3): 404-417.

Martin, Stephen and Peter C. Smith (1999) 'Rationing by waiting lists: an empirical investigation', Journal of Public Economics 71(1): 141-64.

R Development Core Team, R (2010), A Language and Environment for Statistical Computing, R Foundation for Statistical Computing, Vienna, Austria. (Available at $<$ http://www.R-poject.org>.)

Roemer, Milton I. (1961) 'Bed supply and hospital utilization: A natural experiment', Hospitals 35, 988-993.

Sampietro, Laura and Mireia Espallargues (2001) 'Nuevas fórmulas para dar solución a las listas de espera', El Médico, 15 june of 2000: 6064. http://www.diariomedico.com/sanidad/listas/debate.html

Shain, Max and Milton I. Roemer (1959) 'Hospital costs relate to the supply of beds', Modern Hospital 92(4): 71-73.

Siciliani, Luigi and Jeremy Hurst (2003), Explaining waiting times variations for elective surgery across OECD countries, OECD Health Working Papers 7.

Silber Sigmund, H. Mühling, R. Dörr, G. Zindler, A. Preuss and A. Stümpfl (1996) 'Waiting times and death on the waiting list for coronary artery bypass operation. Experiences in Munich with over 1,000 patients', Herz 21(6): 389-396. 
Street, Andrew and Stephen Duckett (1996) 'Are waiting lists inevitable?' Health Policy 36(1): 1-15.

VanBerkel, Peter T. and John T. Blake (2007) 'A comprehensive simulation for wait time reduction and capacity planning applied to general surgery', Health Care Management Science 10(4): 373-385.

Wand, M.P. and M.C. Jones (1995), Kernel Smoothing, Chapman \& Hall, London.

Wilson, J.C. Tunnicliffe (1981) 'Implementation of computer simulation projects in health care', Journal of the Operational Research Society 32(9): 825-832.

Yamaguchi Naoito, Tomohide Tamura, Tomotaka Sobue, Suminori Akiba, Megu Ohtaki, Yoshinobu Baba, Shigeto Mizuno and Shaw Watanabe (1994) 'Evaluation of Cancer prevention strategies by computerized simulation model: Methodological issues', Environmental Health Perspectives 102, Suppl. 8, 64-71.

Zeraati, Hojjat, Farid Zayeri, Gholamreza Babae, Navid Khanafshar and Fatemeh Ramezanzadeh (2005) 'Required hospital beds estimation: A simulation study', Journal of Applied Sciences 5(7): 1189-1191. 